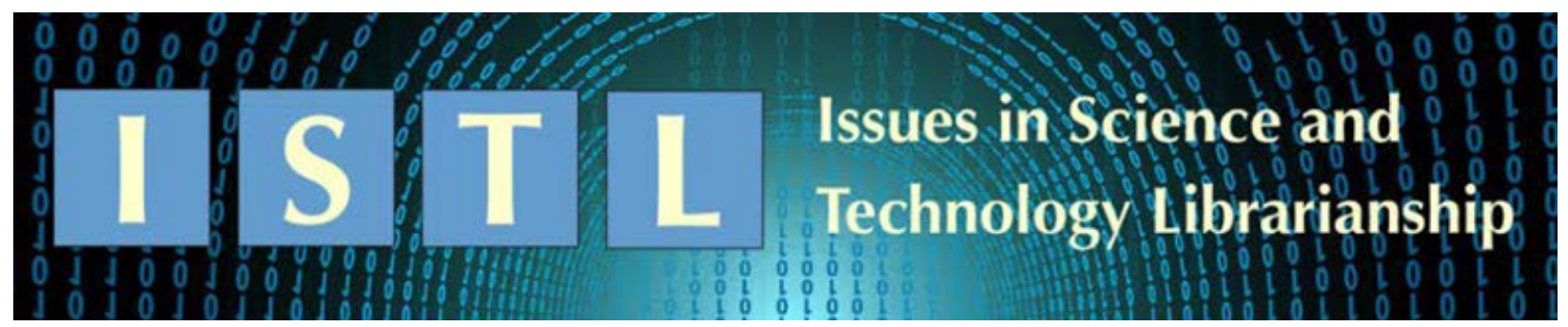

\title{
Launching an Institutional ORCID Initiative at Florida State University
}

\author{
Kelly Grove \\ STEM Research and Learning Librarian \\ Florida State University \\ kegrove@fsu.edu \\ Devin Soper \\ Director of the Office of Digital Research and Scholarship \\ Florida State University \\ dsoper@fsu.edu
}

\begin{abstract}
This case study describes an institutional ORCID initiative at Florida State University. The authors describe how they launched the initiative with minimal resources and staff time at their disposal. The authors also describe specific strategies that can be replicated at other institutions, including identifying the right partners and most compelling use cases, and taking advantage of high-impact outreach strategies that provide the most exposure for the least time invested.
\end{abstract}

\section{Institutional Background}

Florida State University (FSU) is an R1 public research university in Tallahassee, Florida, and has also been home to the National High Magnetic Field Laboratory (MagLab) since 1990. FSU has long prided itself on its "efficiency," and specifically its success in finding savings in areas that do not directly improve the student experience (Irby 2017). In contrast to many R1 institutions (Bryant et al. 2017), then, FSU has not invested heavily in third-party, commercial tools that purport to strengthen the research information management functions of the university. While this institutional context may not sound ripe for an institutional ORCID initiative, the opposite turned out to be true, and the story of how the initiative came to be started at the MagLab.

\section{Why Should Science Librarians Care About ORCID?}

ORCID is a useful tool in the academic publishing world because unique identifiers are used to help distinguish scholars from one another. This is extremely important when more than one person has the same name or as scholars change their names. Researchers in scientific disciplines 
often obtain grants and publish articles at a higher rate than researchers in the humanities and social sciences. With more grants and publications to keep track of, scientists often have the most to gain from using ORCID. As science librarians, it is our duty to help our researchers produce the best possible research and also capture the scope and impact of their work. Additionally, librarians have a role to play in helping their universities stand out from their peers - a challenge that ORCID can help to overcome. If we as librarians bring ORCID integrations into faculty activity reporting workflows on campus, we can help our researchers obtain proper recognition for their work and advance scholarship. As an added perk, campus ORCID initiatives also have the potential to generate new relationships with offices that the library does not normally work with and bring new recognition to the library as a key partner in the research process.

\section{Starting an ORCID Initiative}

In July of 2017, the STEM Libraries were holding their usual office hours out at the MagLab, which is not located on the FSU campus. The MagLab houses the largest and highest-powered magnet laboratory in the world, where thousands of scientists from around the world come to use their equipment and facilities. Normally during these office hours, researchers come to where the librarians sit to ask questions about accessing articles, citation management, or other general library questions. However, on this day the Director of Scientific Instrumentation and Operations at the time came to office hours to ask if the library knew anything about ORCID. This Director oversaw the form scientists had to use in order to request time with the MagLab equipment. The lab's current processes helped them track magnet use time, requests, and users. However, it lacked any capability to track and find any research outputs the users were producing after their time with the equipment. At the time, researchers could request time with the magnets, perform their experiments, take the data, and then were simply asked to report any resulting academic outputs to the MagLab after they left. As it can be imagined, many of the guest researchers did not follow through on reporting their outputs based on the data from their time at the MagLab. This becomes a big issue when it comes to funding reports and applying for new grants. The MagLab could not accurately report the impact their equipment and facilities have on the scientific community because they did not always know the results of all the experiments run by their magnets. Additionally, if the MagLab tried to track down the guest researchers via the names supplied on their request forms, issues of name ambiguity became a problem. Some of the guests had common names and could not be distinguished from their peers. Others published under multiple name variations, making it difficult to connect all their papers back to the same person.

The Director of Scientific Instrumentation and Operations had heard of ORCID but did not know all of the details as to what this type of author identification entailed. He wanted to know if this was a commonly used profile, if they started requiring researchers to have an ORCID when requesting time if it could help them track research outputs, and finally if FSU already had or would consider purchasing an institutional membership to ORCID. It was after hearing about this unique need that the MagLab was trying to fill that the STEM author of this paper became interested in ORCID and how it could help enhance the research at FSU. She took this request to her supervisor and other leaders in the library to start the conversation of ORCID at FSU. During one of these many conversations, it came out that another librarian had tried to generate interest in ORCID, but due to timing and lack of support from other campus organizations the effort was 
set on the back burner. With new interests in ORCID from a major research producing entity on campus, it became easier to start the conversations. With evidence of interest from the MagLab in hand, the then Director of the Libraries' digital scholarship program had everything he needed to approach the Offices of Research and Faculty Development with a proposition to jointly fund an institutional ORCID membership - a proposal which they accepted.

\section{Forming a Team}

After obtaining our institutional ORCID membership, our first task was to form a team to direct and oversee the implementation process. In doing so, we initially set our sights too high, inviting several senior university administrators to serve on an implementation committee. After waiting weeks, sending follow-up emails, and still not hearing back from most of the prospective committee members, we decided to pivot and form an ORCID task force comprised of less senior members. In order for this new task force to be successful, it was important to ensure that the group included adequate representation from all of the relevant stakeholders as well as individuals who had the necessary connections and expertise to undertake the work of ORCID integration. We must have struck the right balance, as our work really took off after we made this switch and recruited the right group of engaged individuals. At FSU, our ideal group included representatives from the Libraries, Faculty Development, IT Services, Research, the MagLab, and the College of Medicine.

\section{Setting Goals}

With the ORCID implementation team in place, our next step was to identify our goals. The obvious goals for our group were related to building integrations between ORCID and key campus systems, on the one hand, and increasing the number of FSU ORCID users, on the other. System integrations can be challenging to implement without extensive support from software engineers, so our goals in this area were modest: namely, to build one integration with our faculty activity reporting system and go from there. With respect to account creation, however, we set our sights fairly high, with the goal of increasing the number of FSU ORCID users by $50 \%$ within one year of obtaining our institutional membership. The plan for reaching this goal involved hosting workshops on ORCID for researchers, partnering with other groups on campus to incorporate ORCID into their workshops and related outreach materials, providing presentations to key stakeholders and university administrators, and, finally, organizing a campus-wide ORCID day that would include workshops and tabling events all over campus. It did not take long to realize that this was going to be way too much for our small group to handle.

\section{Finding the Low-Hanging Fruit}

Recognizing our limited capacity to undertake a sustained, campus-wide program of outreach presentations, we pivoted to instead identify what we believed was the low-hanging fruit for reaching the greatest number of people with the least amount of effort. The first goal in this regard was to integrate ORCID with FSU's homegrown faculty activity reporting system (known as FEAS+). At FSU, all faculty members are required to report their publications, conference presentations, classes taught, and more into FEAS+ in order to be eligible for promotion and tenure. Accordingly, building an integration between ORCID and FEAS+ not only promised to make the process of entering publication data in FEAS+ more efficient and accurate, but also had 
the potential to reach and benefit every faculty member on campus. Indeed, this use case was so compelling that the IT Support Specialist and Lead Developer for FEAS+ prioritized the development of this integration ahead of many other competing priorities. Once this integration was in place, we turned our attention to the question of how to make it as visible as possible to our faculty.

The answer came in the form of the myFSU portal - our institution's centralized, personal gateway to all of the resources that our faculty need for advising, teaching, and administrative functions. By including ORCID prominently in the portal for each employee, we not only reached a vast number of researchers in one stroke, but also created a permanent reminder that researchers can see whenever they log into the portal.

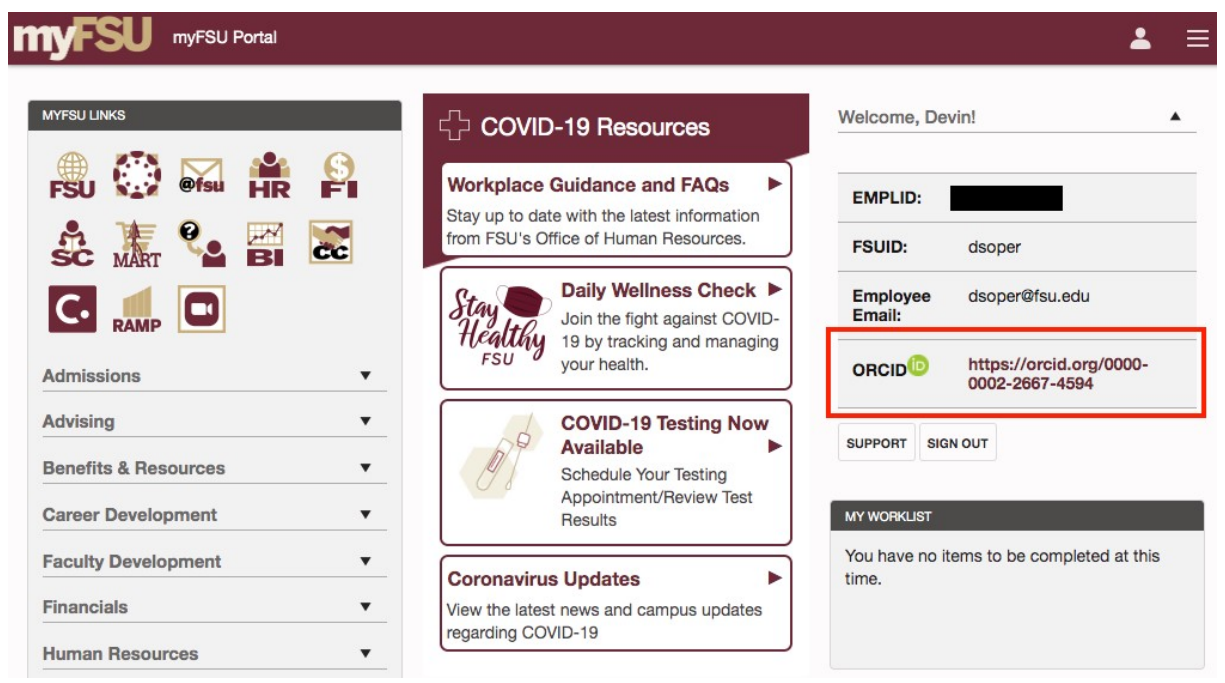

Figure 1. FEAS+ ORCID integration made visible in FSU's central administrative portal for all faculty and staff.

Alongside this effort to make ORCID visible within the myFSU portal, we also worked with our colleagues in Information Technology Services (ITS) to develop a new website (https://orcid.fsu.edu/) with information about ORCID as well as an alternate pathway for researchers to register and connect their ORCID accounts. In addition to the above, we also utilized more conventional outreach strategies such as newsletter announcements and annual workshops at new faculty orientations. However, based on data from ORCID about monthly account registrations, we believe that the myFSU integration was by far the most effective of these strategies; we will discuss this in more detail in the next section.

\section{Measuring Your Impact}

One of the nice things about an ORCID initiative is that it is typically quite easy to measure the impact of the initiative in quantitative terms. Institutional ORCID members receive monthly reports on new ORCID accounts created with institutional email accounts, as well as new and existing ORCID users that have authorized the institution to collect their ORCID ID. 


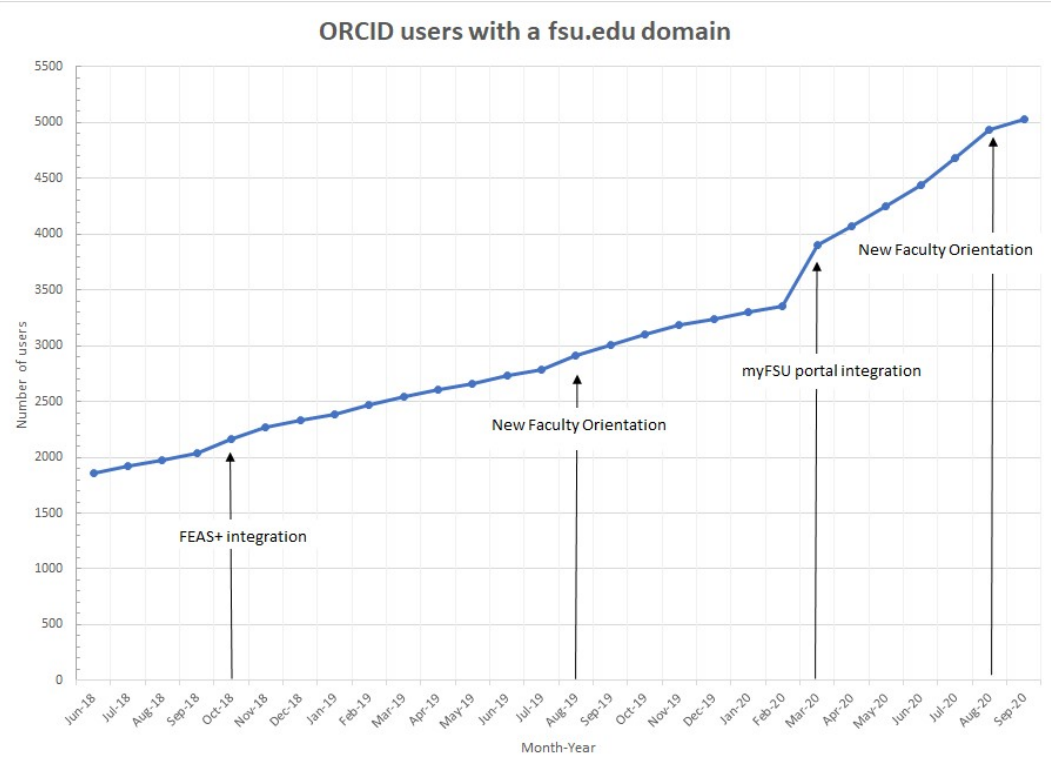

Figure 2. Growth in number of ORCID accounts linked to FSU-affiliated email accounts, with labels for key system integrations and outreach activities.

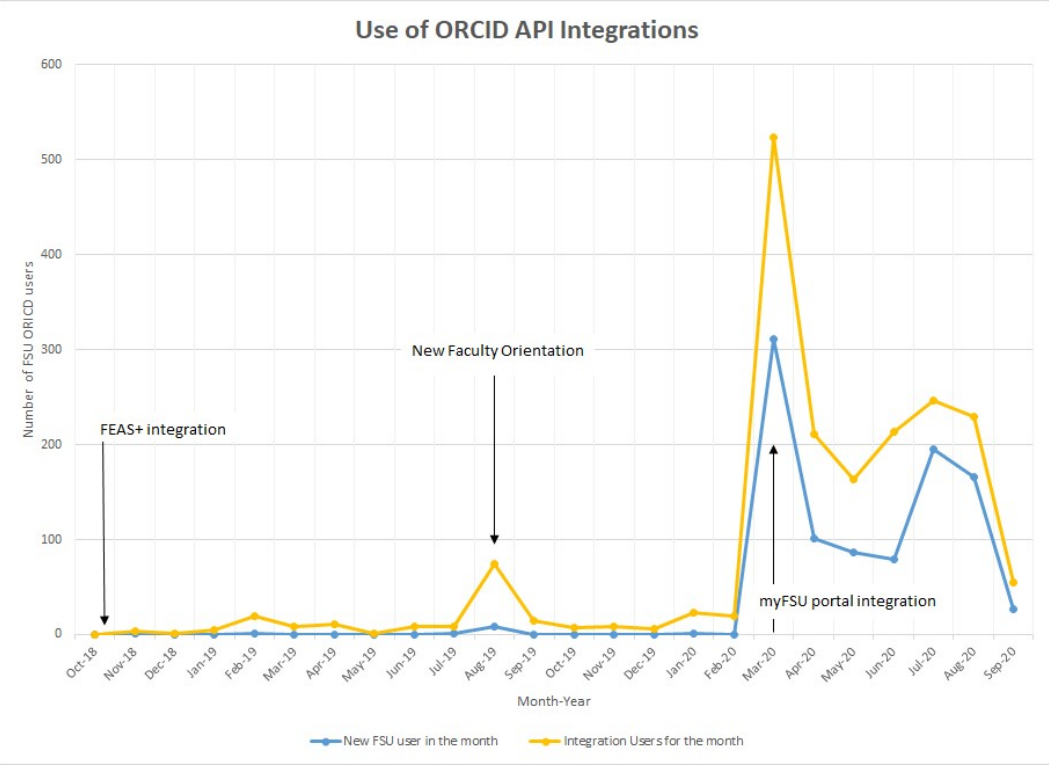

Figure 3. Usage statistics for FSU ORCID system integrations, with labels for key system integrations and outreach activities.

As shown in Figure 2, we experienced steady growth in the number of ORCID accounts linked to FSU-affiliated email addresses from the summer of 2018 to the summer of 2020, with a noticeable increase following the myFSU portal integration described in the previous section. As shown in Figure 3, however, the increase in monthly integration users following the myFSU portal integration was even more dramatic, which suggests that this integration had unprecedented visibility relative to our previous outreach efforts. Overall, we were very satisfied with the rate of growth in new accounts, which rose from 1858 accounts in June 2018 to 5032 accounts in September 2020, an increase of 271\%. Of course, these measures are purely 
quantitative, and we have yet to undertake any qualitative research on the impact of our ORCID initiative. Specifically, we have not asked researchers to explain how they are using the integrations and to what extent they have been helpful for populating data in FEAS+ or other uses. We plan to undertake this additional research in the future.

\section{Conclusion}

We hope that this piece helps to inspire science librarians to consider starting an ORCID initiative at their institutions. Our experience suggests that such initiatives are not difficult to get started when librarians partner with the right people on campus, identify the most compelling use cases, and target the low-hanging fruit that will provide the most impact relative to time invested. As described in the previous section, we were able not only to exceed our goal of increasing total ORCID accounts by $50 \%$ in the first year of our initiative but also sustain that rate of growth in the following year - all without giving up or limiting any of our other duties and responsibilities. Research information management is an increasingly important functional requirement for scientists globally; those based at the NHMFL are by no means an exception in this regard. Academic libraries are well positioned to provide support in this area (Bryant et al. 2017), and science librarians can demonstrate value to the researchers in their subject areas and to their parent institutions by getting involved.

\section{References}

Bryant, R., Clements, A., Feltes, C., Groenewegen, D., Huggard, S., Mercer, H., Missingham, R., Oxnam, M., Rauh A., \& Wright, J. 2017. Research Information Management: Defining RIM and the Library's Role. OCLC Research. DOI: 10.25333/C3NK88.

Irby, K. 2017. Florida State again one of the nation's most efficient universities. Florida State University News. Available from https://news.fsu.edu/news/2017/12/11/florida-state-onenations-efficient-universities/. 